\title{
Polarization attractor optimization for optical signal polarization control
}

\author{
Robert Cybulski $^{1}$ (D) Krzysztof Perlicki ${ }^{1}$
}

Received: 28 February 2018 / Accepted: 10 July 2018 / Published online: 21 July 2018

(c) The Author(s) 2018

\begin{abstract}
Evaluation of polarization control by polarization attractor based on stimulated Raman scattering in optical fiber is presented. Performed simulations are constructed to evaluate polarization control stability for different parameters of polarization attractor: input number of pumps, their power and state of polarization as well as length and nonlinearity of optical fiber. Evaluation of polarization control stability is analogical to crosstalk calculation during the process of splitting two polarization channels with polarization beam splitter. Achieved crosstalk results, supported by degree of polarization calculation, are useful tool for choosing the most optimal setup of polarization attractor elements, depending on the optimization goal.
\end{abstract}

Keywords Nonlinear optics $\cdot$ Raman scattering $\cdot$ Polarization control $\cdot$ Polarization pulling

\section{Introduction}

Polarization of light is one of the basic properties of optical signals, which has been analyzed since the beginning of optical transmission systems (McMaster 1961; Kaminow 1981; Perlicki 2005; Heebner et al. 2000). Although it was investigated carefully both in free space and optical fiber, its application for transmission purposes implies complicated detection methods. This is caused by randomness of optical fibers birefringence, which causes random changes in signal polarization during propagation in the fiber (Agrawal 2012; Boyd 2008). Considering transmission of optical pulses, fiber birefringence influence on signal propagation is represented by PMD (Polarization Mode Dispersion) effect. Commonly used optical fibers are characterized with PMDc (Polarization Mode Dispersion coefficient) of $0.1 \mathrm{ps} / \mathrm{sqrt}(\mathrm{km})$ and lower. This fact makes it impossible to introduce polarization dependence of optical transmission systems, e.g. PDM (Polarization Division Multiplexing), without proper way of polarization control. For obvious reasons basic

Robert Cybulski

R.Cybulski@tele.pw.edu.pl

Krzysztof Perlicki

perlicki@tele.pw.edu.pl

1 Institute of Telecommunications, Warsaw University of Technology, Nowowiejska 15/19,

00-665 Warsaw, Poland 
polarization controllers are not an answer to this problem, as those are entirely passive, mechanically adjusted devices even in case of electrically driven polarization controllers. There is, however, interesting option of polarization control by means of nonlinear polarization attraction in optical fiber. This phenomenon occurs as a result of interaction between signal and stronger pump, where state of polarization of weaker probe signal tends to change towards polarization of the pump. Several different methods of acquiring polarization attraction by exploiting different fiber nonlinear effects were investigated. The most intensive work concerned stimulated Raman scattering (Agrawal and Lin 2003), Brillouin scattering (Thevenaz et al. 2008) and four-wave mixing (Pitois et al. 2008, 2005). Due to the fact that stimulated Raman scattering is commonly exploited in Raman amplifiers, constructing nonlinear polarization attractor based on the stimulated Raman scattering shows potential for polarization control of signals transmitted in different kinds of systems. In particular this concept could be used to introduce PDM to enhance performance of WDM systems (Wavelength Division Multiplexing) or PON (Passive Optical Network) architecture, introducing nonlinear component only in a single point in the system. This is possible if interaction between pump and probe signal occurs in an single device encapsulated fiber, rather than in a transmission fiber. As a form of a step towards polarization attractor construction, author of this paper proposes analysis of polarization attraction efficiency dependence on input pump power, SOP (State of Polarization), fiber length and nonlinearity. This will allow to establish best physical conditions for optical signal polarization control.

This paper is organized as follows: Sect. 2 provides methodology of the research including polarization changes of probe and pump signal as a result of stimulated Raman scattering. In addition this section describes the process of simulation results evaluation. Section 3 presents results and discussion, where Sect. 4 provides conclusion.

\section{Methodology}

\subsection{Pump-signal interaction during stimulated Raman scattering}

The principle of operation of polarization attractor is the interaction between probe signal and stronger pump with proper wavelength during propagation in optical fiber. With proper pump power on the input of the fiber and length of propagation, nonlinear effects such as Raman scattering, SPM (Sef-Phase Modulation) or XPM (Cross-Phase Modulation) must be taken under consideration during signals propagation. Sufficient condition is given by relation between length of the fiber and nonlinear length (Agrawal 2012):

$$
L>\frac{1}{\gamma_{P} P_{0}},
$$

where $\mathrm{L}$ is length of propagation, $\gamma_{P}$ is nonlinear coefficient, at frequency of the pump light and $P_{0}$ is input pump power. Nonlinear coefficient $\gamma_{P}$ is directly connected with real part of third order susceptibility of total electric polarization and is crucial for estimation of nonlinear effects strength.

Stimulated Raman scattering originates from interaction between pump photons, probe signal photons and fiber structure molecules. Incident pump photons scatter on the medium molecules, which results in partial energy transfer from a photon to molecule. This energy is transformed into vibration of the molecule, while the other part of pump photon energy is emitted 
as lower energy Stokes photon. That two-photon generation process is called spontaneous Raman scattering (Stokes photon generation). In the presence of probe signal of frequency lower than pump frequency by the vibrational frequency of medium particles, whole process is stronger by means of difference-frequency generation. In that case probe signal tends to stimulate generation of molecular vibrations, which leads to stronger generation of Stokes photons. Therefore, on the basis of two-photon emission, generated Stokes photon amplifies incident probe signal (Boyd 2008).

Taking that condition (1) is fulfilled and that pump frequency higher than frequency of the probe signal by the vibrational frequency of medium particles, it is possible to observe power transfer from pump to probe signal. In vector analysis one has to additionally consider changes of the pump and signal SOPs during propagation in the fiber. That includes pump-to-signal and signal-to-pump interaction, SPM and XPM induced changes of polarization and the influence of fiber birefringence (Agrawal and Lin 2003):

$$
\begin{gathered}
\frac{d \vec{S}}{d z}=g_{R}\left[(1+3 \mu) P_{0} \vec{S}+(1+\mu) S_{0} \vec{P}-2 \mu S_{3}\right]+\left(\omega_{S} \vec{b}+\overrightarrow{W_{S}}\right) \times \vec{S}-\alpha_{S} \vec{S}, \\
\xi \frac{d \vec{P}}{d z}=\frac{\omega_{P}}{\omega_{S}} g_{R}\left[(1+3 \mu) S_{0} \vec{P}+(1+\mu) P_{0} \vec{S}-2 \mu P_{3}\right]+\left(\omega_{p} \vec{b}+\overrightarrow{W_{P}}\right) \times \vec{P}-\alpha_{P} \vec{P},
\end{gathered}
$$

where $\vec{S}$ and $\vec{P}$ denote probe signal and pump SOPs, $P_{0}$ and $S_{0}$ are initial pump and probe signal optical powers, $S_{3}$ and $P_{3}$ are third coordinates of signal and pump Stokes vectors, $\omega_{S}$ and $\omega_{P}$ denote signal and pump frequencies, $\alpha_{S}$ and $\alpha_{P}$ represent attenuation coefficients at signal and pump frequencies, $\vec{b}$ is the vector of fiber birefringence, $\mu$ is the fiber anisotropy coefficient, $g_{R}$ represents effective Raman gain value for defined frequency shift $(13.2 \mathrm{THz}$ in case of G.652 optical fiber) and $\xi$ equals 1 and -1 for co-propagation and counter-propagation configurations respectively. Vectors $\overrightarrow{W_{S}}$ and $\overrightarrow{W_{P}}$ denote SPM and XPM influence on polarization changes:

$$
\begin{aligned}
& \overrightarrow{W_{S}}=\frac{2}{3} \gamma_{s}\left[S_{3}+2 P_{3}-2 \vec{P}\right], \\
& \overrightarrow{W_{P}}=\frac{2}{3} \gamma_{P}\left[P_{3}+2 S_{3}-2 \vec{S}\right] .
\end{aligned}
$$

Parameter $\gamma_{s}$ is nonlinear coefficient value at probe signal frequency. Analysis of coupled Eqs. (2) and (3) and contribution of their elements to polarization changes was carefully performed by Agrawal and Lin (2003) and Ursini et al. (2011).

Fundamental analysis of $g_{R}$ and $\gamma_{P}$ shows that both depend on the third order susceptibility. Nonlinear parameter $\gamma_{P}$ is described by means of nonlinear refractive index $n_{2}$, frequency of incident pump wave $\omega_{p}$, vacuum speed of light c linear and effective core area $A_{\text {eff }}$ (Agrawal and Lin 2003):

$$
\begin{gathered}
\gamma_{P}=\frac{n_{2} \omega_{p}}{c A_{e f f}}, \text { where } \\
n_{2}=\frac{3}{8 n_{p}} \chi^{\prime(3)} .
\end{gathered}
$$


The form of third order nonlinear susceptibility, represented by $\chi^{(3)}$, depends on whether it is used to describe SPM or XPM. Knowing that SPM is the process caused by selfinduced changes of refractive index, $\chi^{\prime(3)}$ has the form of $\chi_{S P M}^{(3)}=\chi^{(3)}\left(\omega_{s}=\omega_{s}+\omega_{s}-\omega_{s}\right)$, which describes two-photon generation process at probe signal frequency $\omega_{s}$. Similarly $\chi^{\prime(3)}$ for XPM is described by $\chi_{X P M}^{(3)}=\chi^{(3)}\left(\omega_{s}=\omega_{s}+\omega_{p}-\omega_{p}\right)$, as this is a process of Stokes band changes of refractive index induced by incident pump intensity. Analysis of dispersion properties of $\chi^{\prime(3)}$ shows that $\operatorname{Re}\left\{\chi_{X P M}^{(3)}\right\}=0$, therefore:

$$
\gamma_{X P M}=\frac{3 \omega_{p}}{8 n_{p} c A_{e f f}} \operatorname{Im}\left\{\chi_{X P M}^{(3)}\right\} .
$$

Moreover $\operatorname{Im}\left\{\chi_{S P M}^{(3)}\right\}=0$, which implies that:

$$
\gamma_{S P M}=\frac{3 \omega_{s}}{8 n_{s} c A_{e f f}} \operatorname{Re}\left\{\chi_{S P M}^{(3)}\right\}
$$

Nonlinear susceptibility measurements results, demonstrated in by Agrawal and Lin (2003) and Boyd (2008), show that $\operatorname{Im}\left\{\chi_{X P M}^{(3)}\right\}$ and $\operatorname{Re}\left\{\chi_{S P M}^{(3)}\right\}$ are of the same order of magnitude. That implies $\gamma_{X P M} \approx \gamma_{S P M}$ for single mode fibers (Ursini et al. 2011). Peak value of Raman gain factor is represented by analogy to two-photon absorption coefficient $\alpha_{2}$ (Boyd 2008):

$$
g_{R_{\_} \text {peak }}=\frac{3 \omega_{s}}{4 n_{s} c} \chi^{(3)}\left(\omega_{s}=\omega_{s}+\omega_{p}-\omega_{p}\right) .
$$

It is easy to observe that $g_{R_{-} \text {peak }}$ value depends on $\operatorname{Im}\left\{\chi_{X P M}^{(3)}\right\}$ in the same way that $\gamma_{X P M}$. Therefore, as all components of Eq. (2), except attenuation component, depend on multiplication of pump power and $\chi^{(3)}$, author proposes to evaluate polarization attraction effectiveness with respect to $P_{0} \cdot \gamma_{X P M}$. This approximation may allow to analyze process of polarization control for optical fibers with different nonlinearity level.

\subsection{Methodology of polarization control stability evaluation}

In order to analyze the effectiveness of polarization pulling one has to consider different efficiency of polarization changes and power transfer for different relative SOPs, as was shown in previous analysis (Agrawal and Lin 2003). Therefore author proposes to perform analysis for a number of input probe signal SOPs, evenly distributed on Poincaré sphere (presented on Fig. 1) and to evaluate the degree of probe signal SOPs aggregation on the Poincaré sphere. This can be done through calculation of angle between particular probe signal SOP and specific point of Poincare sphere, which is the center point of probe signal SOPs cluster. Figure 2 presents group of Poincaré spheres for different input pump powers, left circular input pump SOP and $15 \mathrm{~km}$ standard single mode fiber, which show probe signal SOPs after the process of polarization attraction. Each sphere presents also center point of probe signal SOPs cluster marked with a X sign. That way it is possible to calculate maximum value of such angle for all probe signal SOPs at each polarization attractor setup (input pump power and SOP and length of the fiber). 
Fig. 164 equally distributed input probe signal SOPs
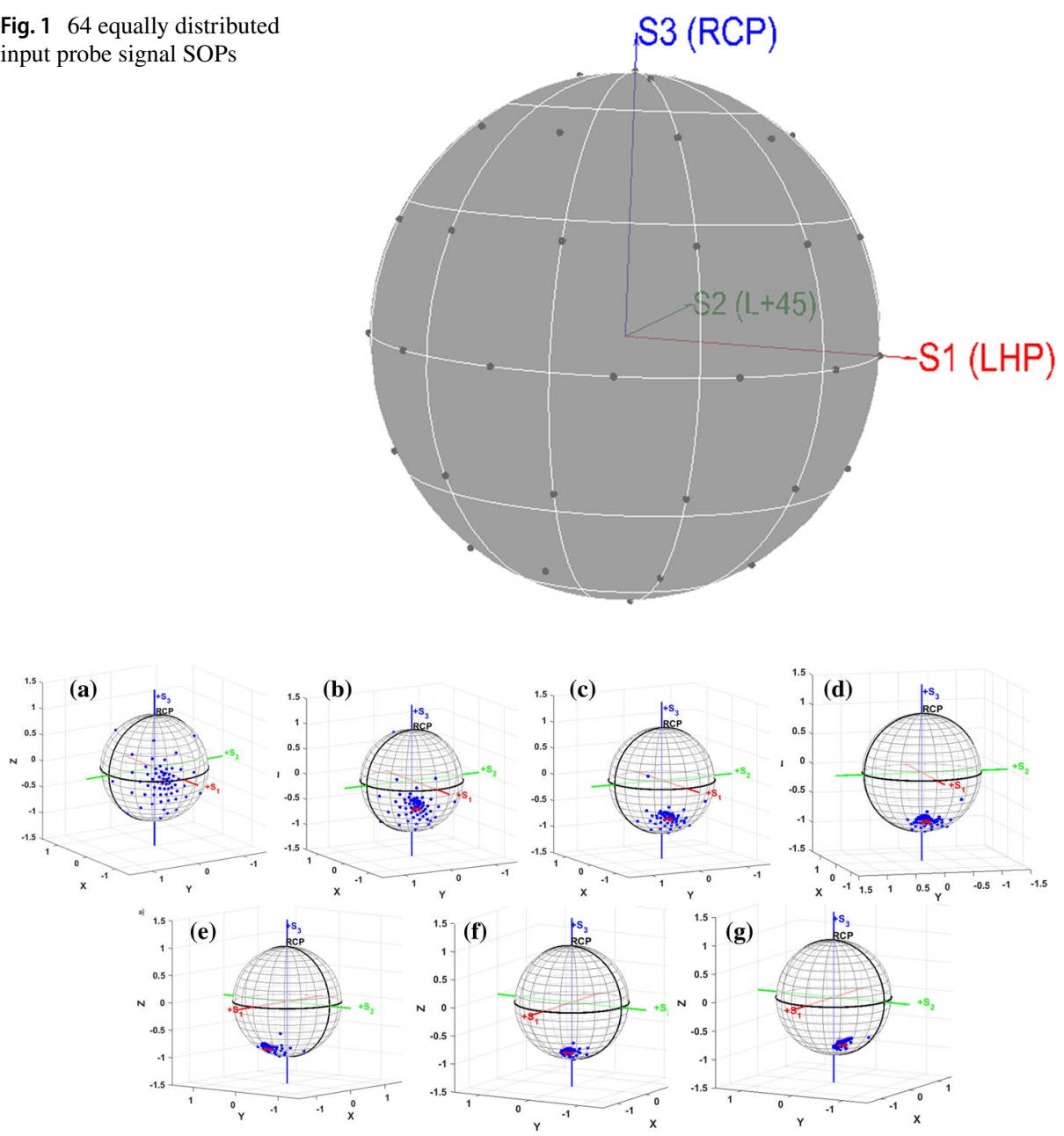

Fig. 2 Probe signal SOPs evolution at the end of $15 \mathrm{~km}$ standard single mode fiber for left circular input pump SOP and input pump powers: a $0.9 \mathrm{~W}$, b $1 \mathrm{~W}$, c $1.1 \mathrm{~W}, \mathbf{d} 1.2 \mathrm{~W}$, e $1.3 \mathrm{~W}, \mathbf{f} 1.4 \mathrm{~W}$, g $1.5 \mathrm{~W}$. X marks median of all probe signal SOPs

Although angle evaluation is universal metric, it has no direct connection to transmission systems requirements like inter-channel crosstalk in WDM systems. That is why author proposes analogical approach to the one presented by Rochat et al. (2004), where crosstalk between two polarization channels in the process of separating them in PBS (Polarization Beam Splitter) is analyzed. Crosstalk was calculated as dependent on the angle between two SOPs (corresponding to polarization channels) in the plane created by S1 and S2 axis of Poincare sphere. Situation is shown on Fig. 3. Angle $\theta$ is the angle between two SOPs and $\varphi$ denotes angle between bisector of realigned SOPs and axis of PBS. PBS X-axis is aligned with S1 axis and Y-axis is oriented along S2 axis of Poincare sphere. Therefore crosstalk is defined as:

$$
X_{t x}=X_{t y}=20 \log \left(\tan \left(45^{\circ}-\frac{\theta}{2}\right)\right)
$$


Fig. 3 Projections of two SOPs: $\mathrm{Sa}$ and $\mathrm{Sb}$ on the plane created by PBS axis. (Reproduced with permission from Rochat et al. 2004)

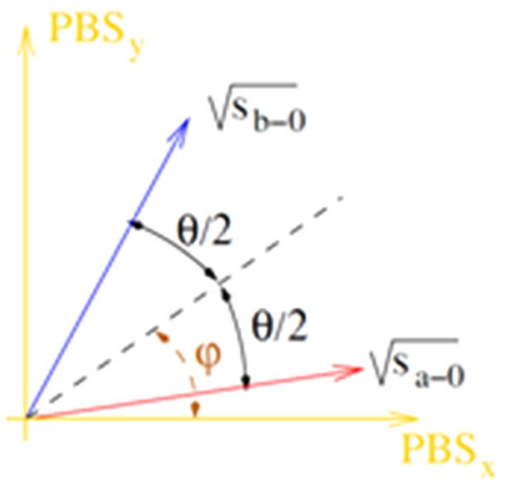

Equation (11) is correct taking that $\varphi=45^{\circ}$ (Rochat et al. 2004).

Author proposes to use similar approach and use the crosstalk calculation for polarization control stability evaluation. For this purpose one can analyze spreading of the cluster of points on the Poincare sphere, by means of maximum value of the angle between particular SOP and a median of SOPs- $\alpha$. This procedure leads to number of angles values, which can be converted to crosstalk values. To do so single probe signal SOP $\vec{S}$ can be concerned as a combination of orthogonal SOPs $\left(\theta=90^{\circ}\right)$. To calculate crosstalk for the orthogonal components of $\vec{S}$, for polarization control stability evaluation, one needs to align set of vectors $\vec{S}$ and median SOP, so that median SOP is aligned with bisector of PBS axis system. This is a safe operation as it can be done by exploiting QWPs (Quarter-Wave Plate) and HWP (Half-Wave Plate), which preserves $\alpha$ angle between vectors. Situation is presented on Fig. 4. Angle $\alpha$ is also angle between particular SOP and bisector of PBS axis system, therefore one may define relation between $\alpha$ and crosstalk for $\vec{S}$ components, when splitting them by means of PBS:

$$
X_{t x}=X_{t y}=20 \log (\tan (\alpha)) \text {. }
$$

Fig. 4 Angle between $\vec{S}$ and $\overrightarrow{\text { medianSOP}}$ on the PBS plane

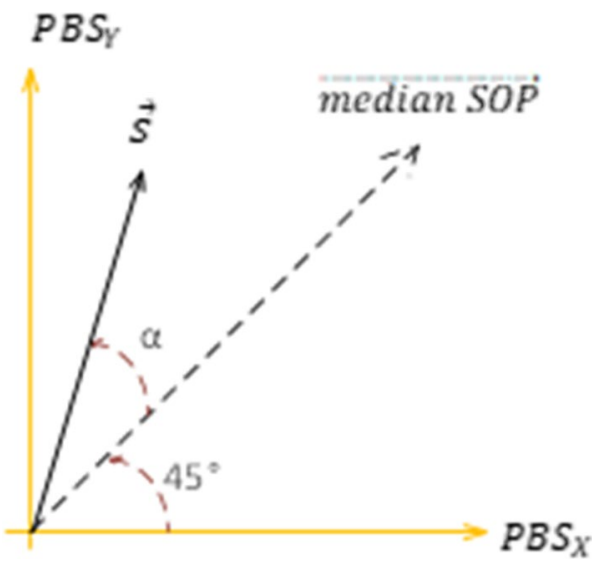




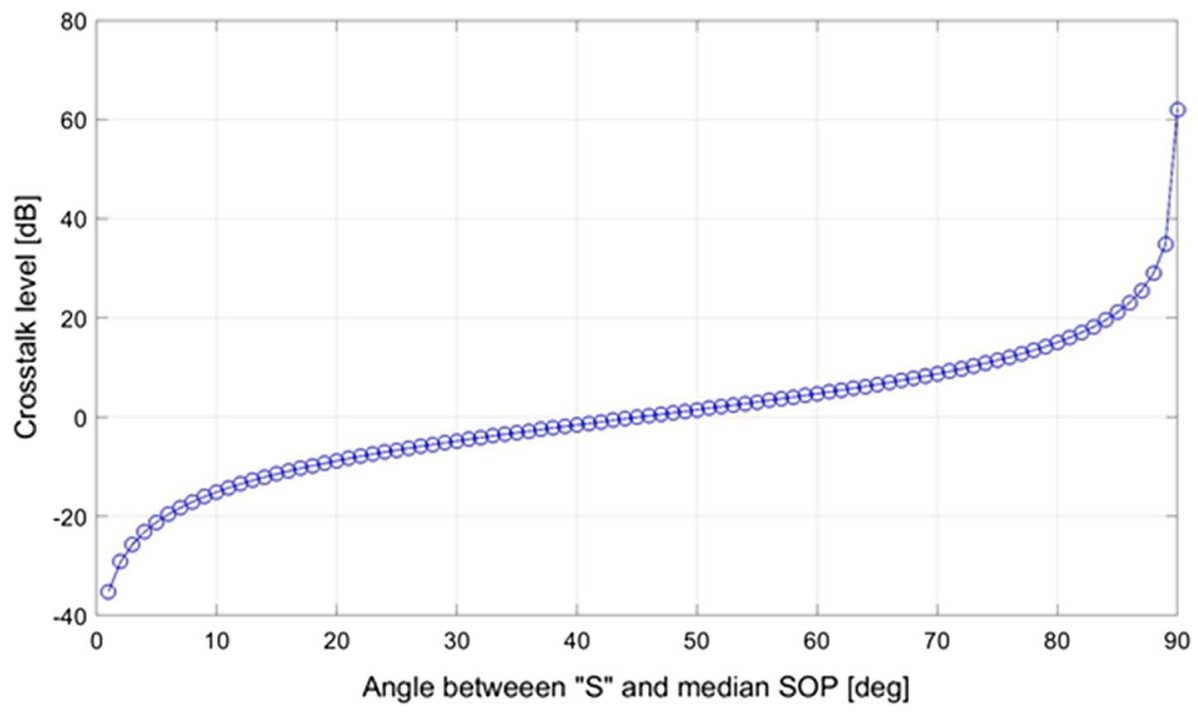

Fig. 5 Crosstalk level between orthogonal components of $\vec{S}$ versus angle between $\vec{S}$ and median SOP, caused by splitting $\vec{S}$ components by PBS ("S" denotes vector $\vec{S}$ )

Fig. 6 Scheme of polarization attractor in co-propagating configuration. LP block denotes pump laser, $\mathrm{PC}$ is the polarization controller, $\mathrm{C}$ is optical combiner, $\mathrm{R}$ is rotation elements block, containing QWP and/ or HWP. A and B denote input and output of the polarization attractor

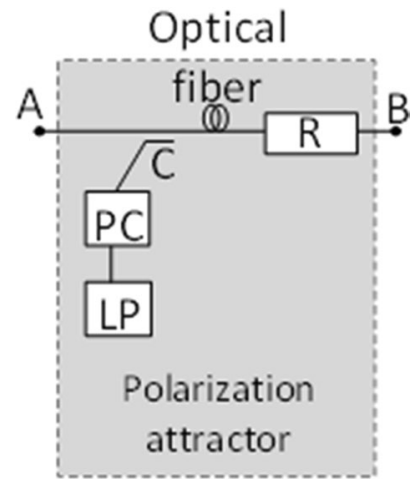

As operation of rotation of $\vec{S}$ and median SOP preserves angle $\alpha$, calculation of crosstalk may be performed without rotating vectors previously. Figure 5 present calculation of crosstalk for $\alpha$ in range $0^{\circ}-90^{\circ}$. It is easy observe that the larger is the angle $\alpha$, the higher is the level of crosstalk between orthogonal components of $\vec{S}$ as a result of splitting them by PBS. For $\alpha$ values higher than $45^{\circ}$ crosstalk level exceeds $0 \mathrm{~dB}$, which means that particular component of $\vec{S}$ is received on the wrong axis of PBS. Therefore polarization attraction process must be effective enough to ensure $\alpha$ angle values below $45^{\circ}$.

\subsection{Polarization attractor scheme for polarization control}

Figure 6 shows block scheme of polarization attractor. Probe signal SOP at the input point A is random (Fig. 1). It is combined with the pump laser, which SOP is previously 
controlled in polarization controller. Polarization attraction and amplification due to stimulated Raman scattering occurs in optical fiber. Afterwards probe signal SOP is constant, but not equal to initial pump SOP, as described in Sect. 2.1. In order to separate orthogonal components of signal SOP, with minimal crosstalk, one may have to use QWP and/or HWP (R block) to align signal SOP with PBS axis system.

\subsection{Simulation scenarios and parameters}

Simulation software chosen for the purpose of analysis polarization attraction effectiveness is VPItranssmissionMaker Optical Systems by VPIphotonics. Due to the fact of random nature of optical fiber birefringence, it is essential that process of polarization control stability estimation includes statistical analysis. In this case author proposes to analyze 64 equally Poincare sphere-distributed SOPs of probe signal, at $1550 \mathrm{~nm}$, in each simulation. Moreover as the process of polarization changes depends on input pump power and length of the fiber, input CW (Continuous Wave) pump power, at $1457 \mathrm{~nm}$, is changed from $0.9-1.5 \mathrm{~W}$ and fiber length changes in the range of 10-17 km. Fiber type is standard single mode fiber G.652 for which nonlinear coefficient $\gamma_{X P M}$ at $\omega_{s}=2 \pi \cdot 206.3 \mathrm{THz}$ is approximately equal $1 /(\mathrm{W} \mathrm{km})$ according to Agrawal and Lin (2003).

Both co-propagating and counter-propagating configurations of the pump and probe signal are verified with PMDc of the fiber set to $0.01 \mathrm{ps} / \mathrm{sqrt}(\mathrm{km})$. Choice of PMDc value is crucial for polarization stability and amplification of probe signal. Polarization pulling will be most effective, when beat length of the fiber is much larger than correlation length, which was investigated by Martinelli et al. (2009). It can be seen that for PMDc values greater than $0.01 \mathrm{ps} / \mathrm{sqrt}(\mathrm{km})$, changes of input pump power do not cause any impact on relative angle between pump and probe signal SOPs. For this PMDc value beat length of the fiber is larger by an order of magnitude than correlation length.

In order to find optimal set of polarization attractor elements parameters, author proposes to verify dependence of polarization pulling effectiveness on input SOP of the pump. Input pump SOPs chosen for analysis are: horizontal, linear $+45^{\circ}$ and left circular polarization.

\section{Results and discussion}

This section provides presentation of simulation results, in which co-propagating pump is used to observe polarization control of probe signal by polarization attraction.

\subsection{Co-propagation configuration results}

Effectiveness of polarization pulling process depends on the input length of the fiber, $P_{0} \gamma$ and pump SOP, which is why Fig. 7 shows maximum value of crosstalk level for every simulation configuration. Maximum crosstalk value is obtained from crosstalk values for all probe signal SOPs. Crosstalk level is calculated according to Eq. (12). Decision to analyse maximum values of angle and crosstalk is justified by the fact of poor efficiency of power transfer from pump to probe signal in case of orthogonal relation of the SOPs. This interaction efficiency is approximately lower by half than in case of parallel SOPs of probe signal and pump (Agrawal and Lin 2003; Agrawal 2012). It can be also seen on the Fig. 2, that some probe signal SOPs are attracted slower than the rest. These SOPs, which are 


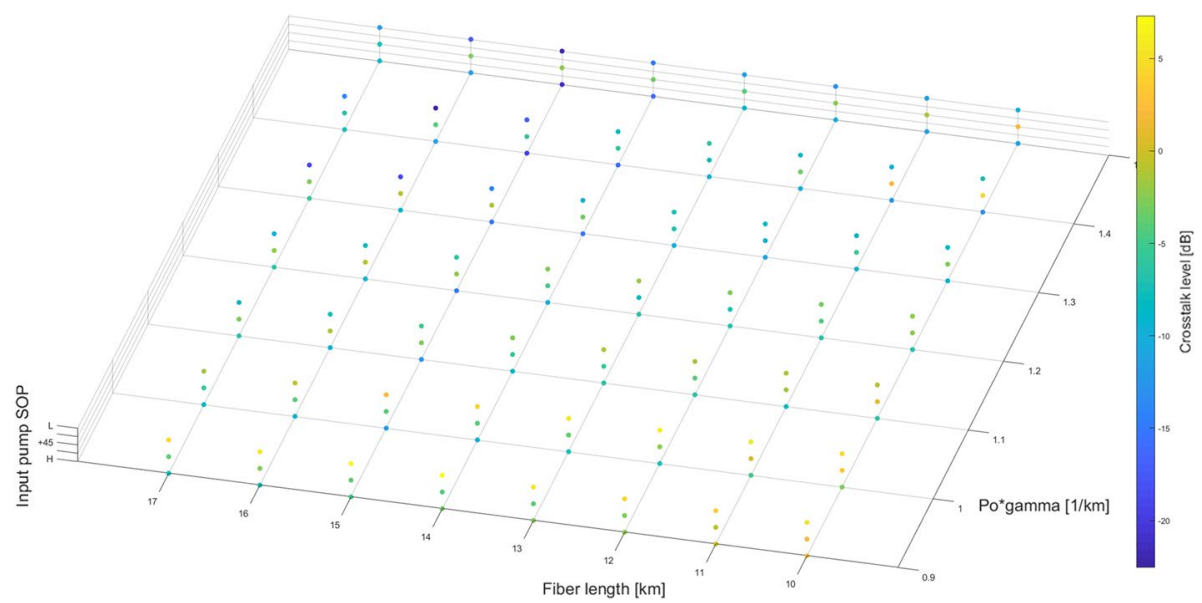

Fig. 7 Maximum crosstalk level between orthogonal components of $\vec{S}$ versus angle between $\vec{S}$ and median SOP, caused by splitting $\vec{S}$ components by PBS for different parameters of polarization attractor ("S" denotes vector $\vec{S}$ ) and co-propagation configuration. SOPs description: L: left circular, $+45-$ linear $+45^{\circ}$, $\mathrm{H}-$ horizontal

attracted most slowly, were initially orthogonal to the pump SOP. Analysis of Fig. 7 presents that larger $P_{0} \gamma$ values provide more effective aggregation of probe signal SOPs cluster on Poincaré, which results in lower crosstalk levels. This fact can be observed for every fiber length. Table 1 presents simulation results for several configurations, which provide the lowest crosstalk levels. As one can observe left circular and horizontal input pump SOPs are most suitable, when considering crosstalk level minimization. The best results are obtained for $16 \mathrm{~km}$ fiber and $P_{0} \gamma$ value of $1.41 / \mathrm{km}$, which guarantee crosstalk level of $-22.5 \mathrm{~dB}$. Crosstalk level of $-20 \mathrm{~dB}$ is approved as maximum crosstalk level between channels in WDM systems (Perlicki 2012), therefore in present work it is considered as reference maximum acceptable crosstalk value. Similar investigation was performed by Martinelli et al. (2009). In spite of the fact, that simulated fiber was $2 \mathrm{~km}$ dispersion shifted and pump powers were much higher, comparison can be found by calculating the value of $L P_{0} \gamma$. In discussed case this value is higher than $20(\gamma>1)$ for $8 \mathrm{~W}$ pump power, which allows to control polarization of probe signal, independently on input probe signal SOP. Similar level of probe signal polarization control was obtained in present work for $15 \mathrm{~km}$ standard single mode fiber, $1.5 \mathrm{~W}$ of input pump power and left input pump SOP (Fig. $2 \mathrm{~g}$ ).

For complete analysis of polarization changes probe signal DOP calculation is necessary. Figures 8, 9 and 10 present DOP calculation for left circular, linear $+45^{\circ}$ and

Table 1 List of configurations with the lowest crosstalk levels for pump and probe signal co-propagating configuration

\begin{tabular}{llllll}
\hline $\begin{array}{l}\text { Configuration } \\
\text { no. }\end{array}$ & Input pump SOP & $P_{0} \gamma(1 / \mathrm{km})$ & $\begin{array}{l}\text { Fiber length } \\
(\mathrm{km})\end{array}$ & $\begin{array}{l}\text { Crosstalk level } \\
(\mathrm{dB})\end{array}$ & DOP (\%) \\
\hline 1 & Left circular & 1.4 & 16 & -22.5 & 99.81 \\
2 & Left circular & 1.5 & 15 & -21.9 & 99.85 \\
3 & Horizontal & 1.5 & 15 & -20.9 & 98.54 \\
\hline
\end{tabular}




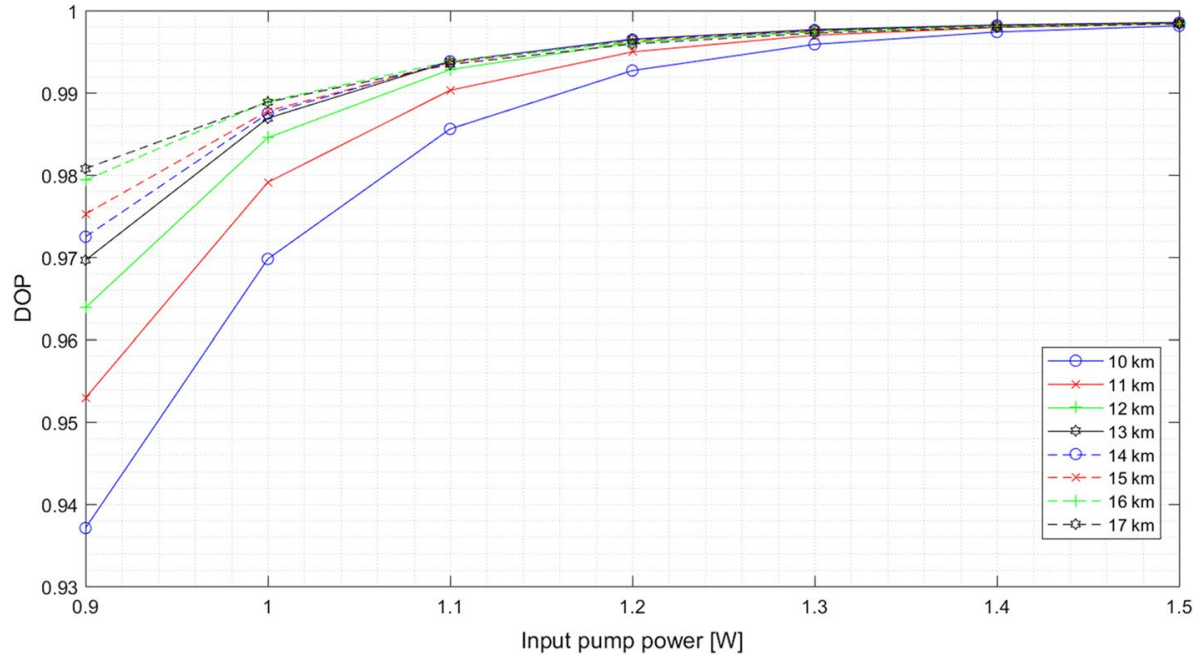

Fig. 8 Degree of polarization of probe signal after polarization pulling for left circular input pump SOP, all input pump powers and fiber lengths (co-propagation configuration)

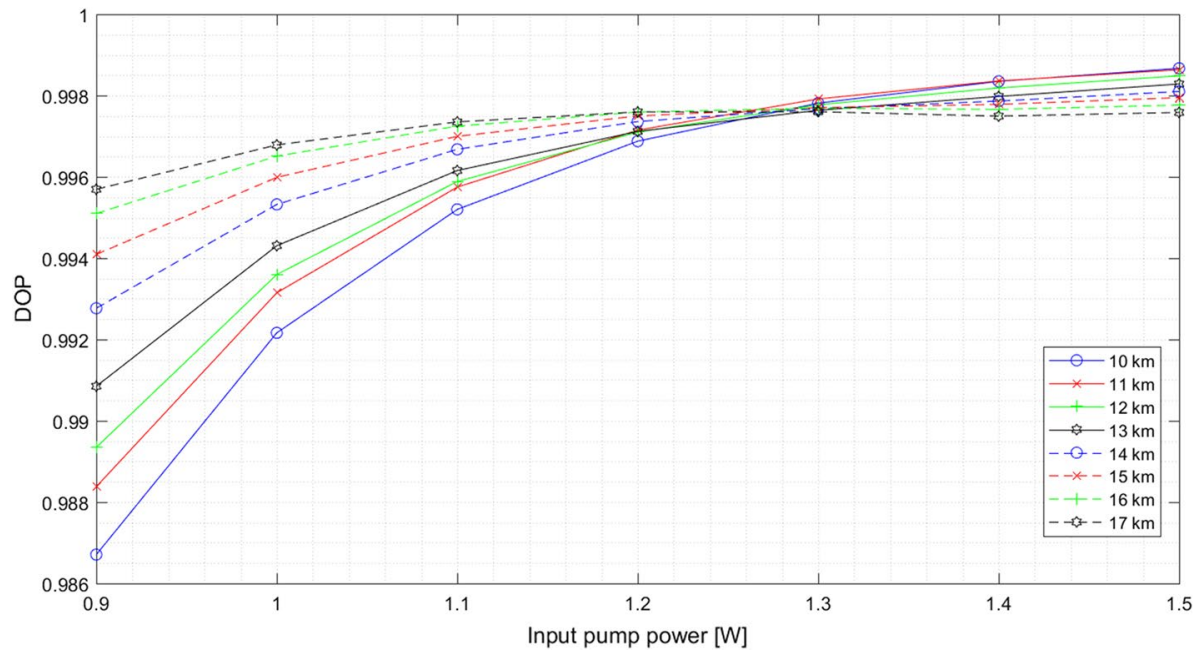

Fig. 9 Degree of polarization of probe signal after polarization pulling for linear $+45^{\circ}$ input pump SOP, all input pump powers and fiber lengths (co-propagation configuration)

horizontal input pump SOPs respectively. Similarly to the case of polarization control, growing input pump power results in higher probe signal DOP values. For left circular pump SOP it can be observed that longer fiber causes faster saturation of DOP characteristics, however for $P_{0} \gamma$ values greater than $1.21 / \mathrm{km}$ and fibers longer than $10 \mathrm{~km}$ those differences become neglible (Fig. 8). In this range DOP remains stable above $99 \%$. Change of input pump SOP to linear $+45^{\circ}$ results in DOP values higher by approximately $1.5 \%$, however for fibers longer than $13 \mathrm{~km}$ and $P_{0} \gamma$ greater than $1.31 / \mathrm{km}$ probe signal DOP is 


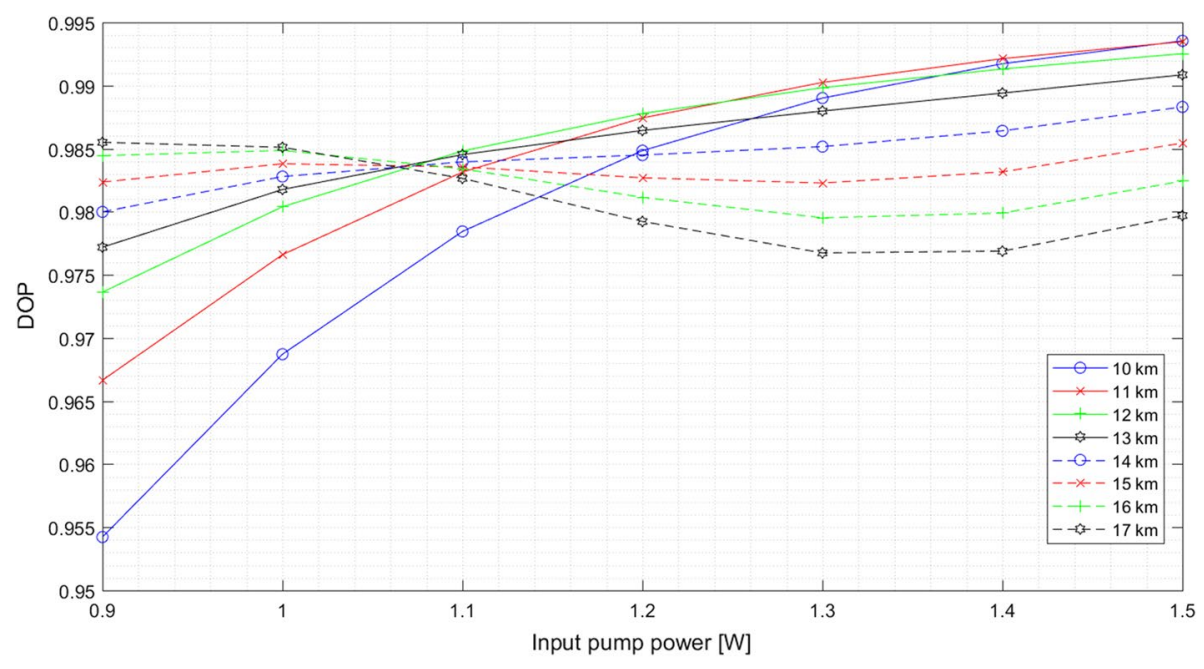

Fig. 10 Degree of polarization of probe signal after polarization pulling for horizontal input pump SOP, all input pump powers and fiber lengths (co-propagation configuration)

not enhanced by polarization pulling as effectively as for shorter fibers (Fig. 9). This effect is even stronger for horizontal input pump SOP. Fibers longer than $13 \mathrm{~km}$ and $P_{0} \gamma$ greater than $1.11 / \mathrm{km}$ cause that probe signal DOP is evidently decreased to $97 \%$ (Fig. 10). This degradation is the effect of nonlinear polarization rotation, induced by cross phase modulation. Decrease of DOP value would be even greater, if it was not for pump depletion, which mitigates the influence of NPR. This fact was previously verified for significantly higher pump powers and different fiber PMDc values (Ursini et al. 2011). Analysis of these results shows that for PMDc value of $0.01 \mathrm{ps} / \mathrm{sqrt}(\mathrm{km})$ probe signal DOP can decrease even to 0.5 for pump power of $14 \mathrm{~W}$. DOP degradation presented in this paper is lower, because of lower pump power, but it is still possible to notice that DOP decreasing depends on input pump SOP. Taking that, configurations exploiting left circular input pump SOP are potentially more attractive, when simultaneous crosstalk level and probe signal DOP values are to be controlled. For configurations presented in Table 1 probe signal DOP remains above 98\%, which can be compared to results performed by Muga et al. (2011). Co-propagation polarization pulling in $2 \mathrm{~km}$ optical fiber with $\gamma=21 /(\mathrm{W} \cdot \mathrm{km})$ and input pump power up to $12 \mathrm{~W}$ was presented. For $6 \mathrm{~W}$ input pump power, which resulted in $L P_{0} \gamma$ value of 24 , calculated probe signal DOP was equal to approximately $97 \%$. Results of polarization pulling in $2 \mathrm{~km}$ optical fiber with $\gamma=11 /(\mathrm{W} \cdot \mathrm{km})$ and input pump power up to $20 \mathrm{~W}$, presented by Ursini et al. (2011) are also comparable with present results. For input pump power of $10 \mathrm{~W}$ and PMDc value of $0.01 \mathrm{ps} / \mathrm{sqrt}(\mathrm{km})$ DOP value remains close to $100 \%$.

\subsection{Counter-propagation configuration results}

In case of counter-propagation configuration, similarly to the case of co-propagation simulation results, growing $P_{0} \gamma$ values cause stronger probe signal SOPs aggregation due to the polarization pulling effect. This fact can be observed for every fiber length (Fig. 11). However in the case of counter-propagating pump and probe signal only one polarization attractor configuration can provide crosstalk level below $-20 \mathrm{~dB}$. This occurs for $14 \mathrm{~km}$ 


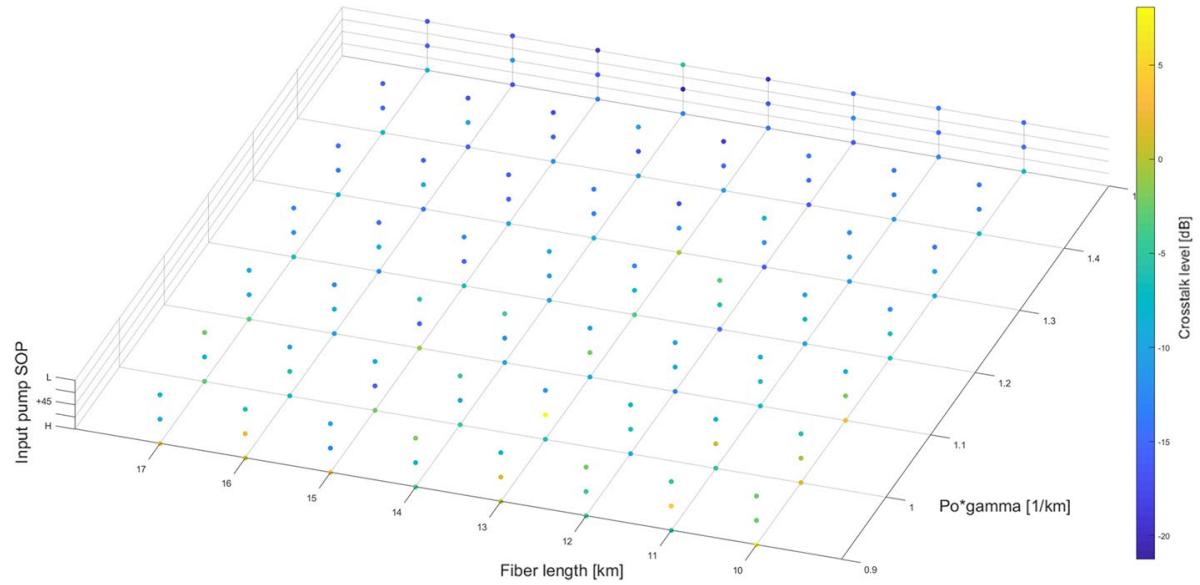

Fig. 11 Maximum crosstalk level between orthogonal components of $\vec{S}$ versus angle between $\vec{S}$ and median SOP, caused by splitting $\vec{S}$ components by PBS for different parameters of polarization attractor ("S" denotes vector $\vec{S}$ ) and counter-propagation configuration. SOPs description: L: left circular, +45-linear $+45^{\circ}, \mathrm{H}$-horizontal

fiber with $1.51 / \mathrm{km} P_{0} \gamma$ value. Probe signal DOP analysis shows that in case of input left circular pump SOP, the longer is the fiber the flatter is DOP curve. For $17 \mathrm{~km}$ fiber DOP values remain nearly constant for all $P_{0} \gamma$ values (Fig. 12). For linear $+45^{\circ}$ input pump SOP flat DOP curves are obtained for fiber longer than $15 \mathrm{~km}$, however tendency, observed for left input pump SOP, is disturbed as DOP curve for $16 \mathrm{~km}$ fiber presents strong $P_{0} \gamma$ dependence (Fig. 13). Horizontal input pump SOP configuration exhibits no significant differences of DOP values for different fiber lengths (maximum difference for $P_{0} \gamma$ value

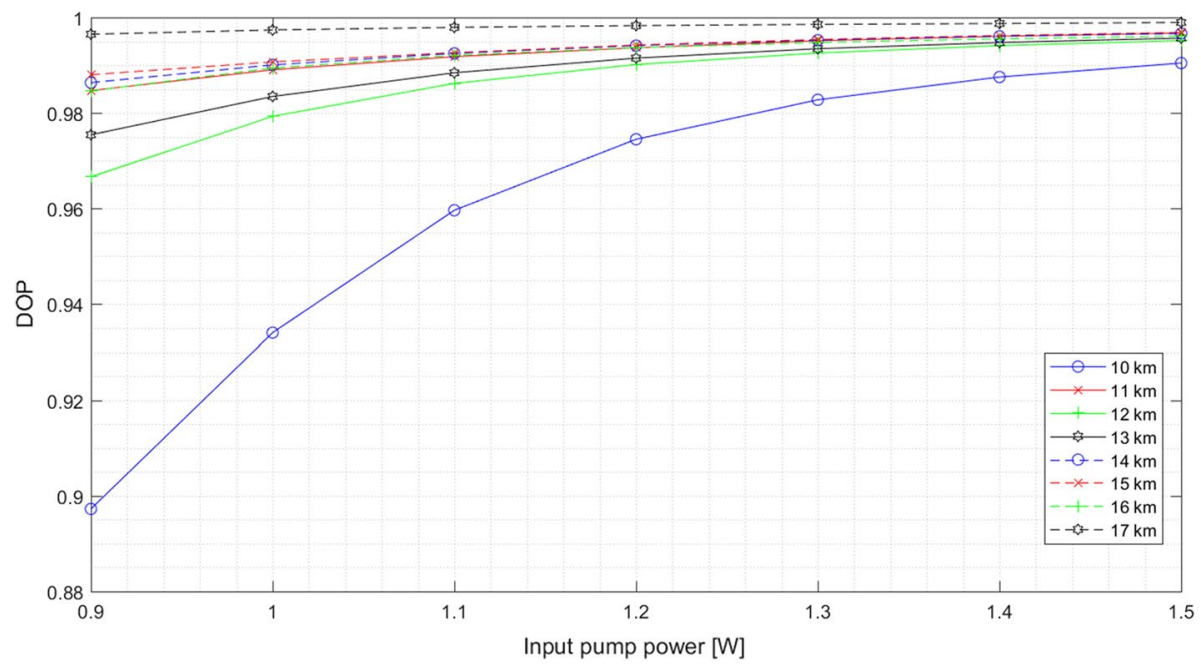

Fig. 12 Degree of polarization of probe signal after polarization pulling for left circular input pump SOP, all input pump powers and fiber lengths (counter-propagation configuration) 


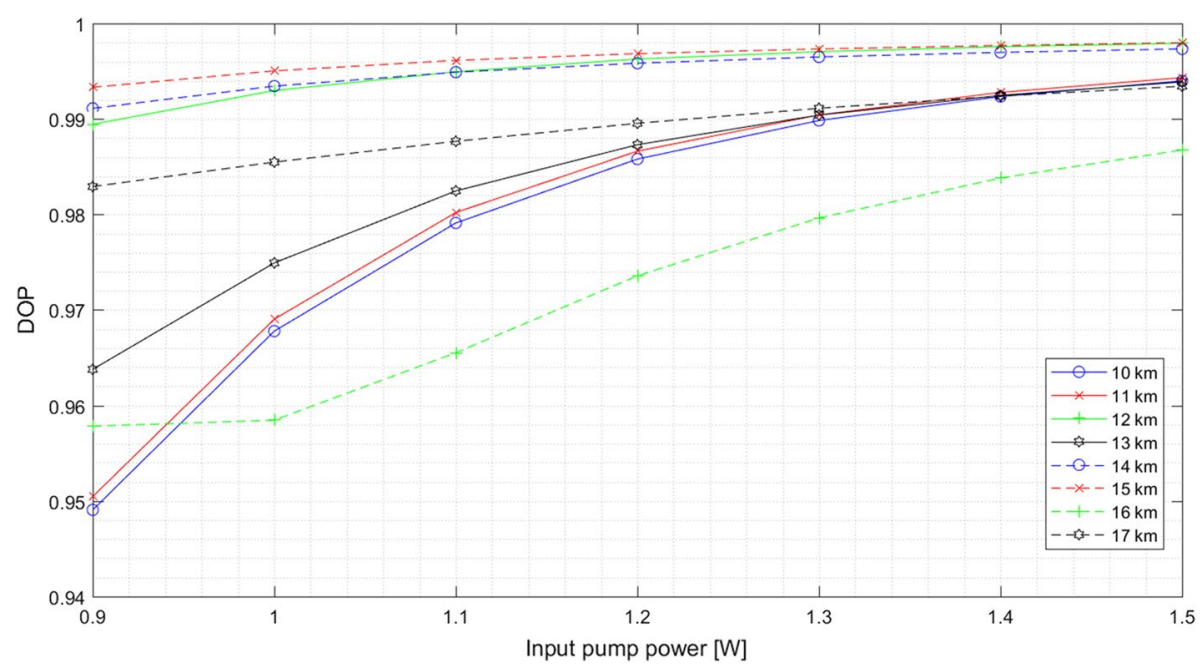

Fig. 13 Degree of polarization of probe signal after polarization pulling for linear $+45^{\circ}$ input pump SOP, all input pump powers and fiber lengths (counter-propagation configuration)

of $0.91 / \mathrm{km}$ is lower than $0.5 \%$ ), with simultaneous flat DOP curves (Fig. 14). However due to the fact of crosstalk levels higher than $-20 \mathrm{~dB}$, configurations exploiting horizontal input pump SOP cannot be taken under consideration, when choosing optimum polarization attractor setup. For $14 \mathrm{~km}$ fiber, $1.51 / \mathrm{km} P_{0} \gamma$ value and linear $+45^{\circ}$ input pump SOP, DOP value is approximately $99.5 \%$. This results are comparable with DOP calculation presented by Chiarello et al. (2011), where counter-propagation configuration simulation of polarization pulling with $2 \mathrm{~km}$ standard single mode fiber and input pump powers up

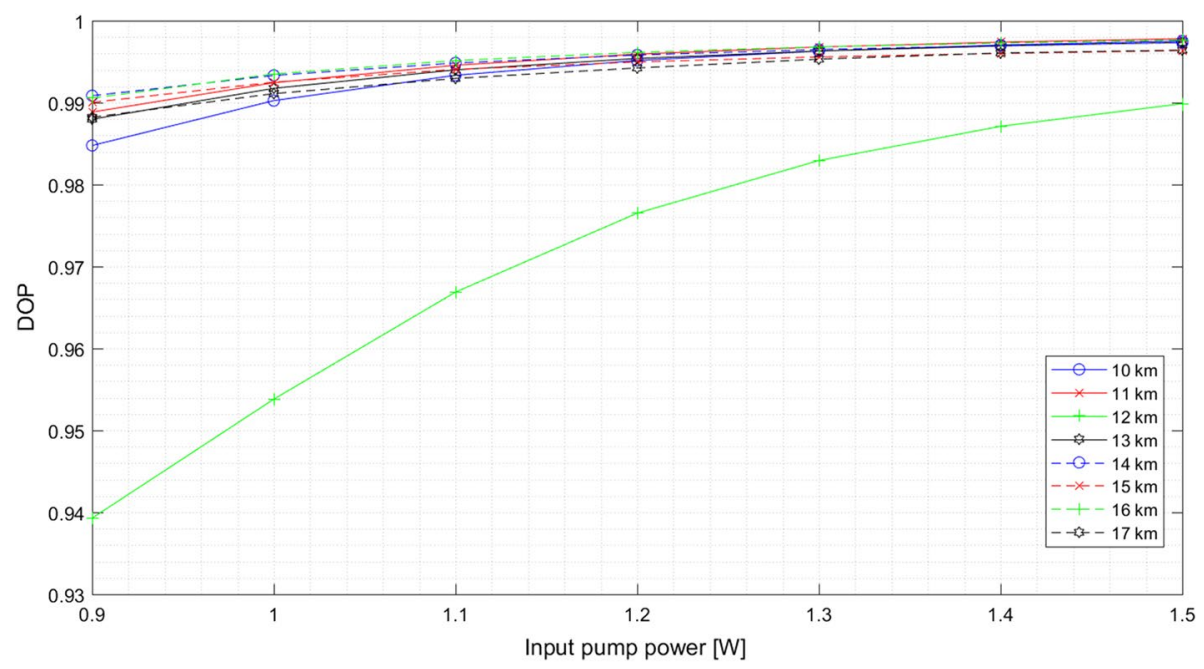

Fig. 14 Degree of polarization of probe signal after polarization pulling for horizontal input pump SOP, all input pump powers and fiber lengths (counter-propagation configuration) 
to $10 \mathrm{~W}$ was performed. It can be noticed that for PMDc value of $0.01 \mathrm{ps} / \mathrm{sqrt}(\mathrm{km})$ and $10 \mathrm{~W}$ of input pump power $\left(L P_{0} \gamma=20\right)$ DOP value is higher than $90 \%$. DOP values close to unity for counter-propagation polarization pulling in strong pump depletion regime with $L P_{0} \gamma=20.4$ were also presented by Morin et al. (2012) on Fig. 6.

Comparing results for both propagations in depleted pump regime and PMDc value of $0.01 \mathrm{ps} / \mathrm{sqrt}(\mathrm{km})$ shows that counter-propagation of pump and probe signal provides weaker aggregation of probe signal SOPs on Poincarè sphere and higher crosstalk levels, which is proved by only one configuration with crosstalk level below $-20 \mathrm{~dB}$ for counter-propagation configuration. However, one can observe that for fiber longer than $14 \mathrm{~km}$ and $P_{0} \gamma$ higher than $1.21 / \mathrm{km}$ differences between crosstalk levels for different input pump SOPs are lower than in case of co-propagating configurations. That is why, although there are several polarization attractor set-ups exploiting pump and signal co-propagation with crosstalk lower than $-20 \mathrm{~dB}$, they require more precision with input pump SOP setting. On the other hand DOP analysis shows that pump and signal counter-propagation vanishes the effect of DOP decreasing, which is clear for co-propagation configurations, especially for horizontal and linear $+45^{\circ}$ input pump SOPs. This fact makes it attractive to use counterpropagation polarization attractor configuration for probe signal polarization control, when considering optimal, $P_{0} \gamma$ independent DOP characteristics. Moreover, as shown by Chiarello et al. (2011), counter-propagation configurations offer stable and high DOP values for PMDc values even higher than $0.01 \mathrm{ps} / \mathrm{sqrt}(\mathrm{km})$ for high input pump power. This is not observable for co-propagation configurations, as described by Ursini et al. (2011).

\section{Conclusion}

Presented idea exploits stimulated Raman scattering in optical fiber to obtain all-optical polarization control. Author proposes to evaluate probe signal polarization control stability by means of angular and crosstalk analysis. Crosstalk calculation is based on signal orthogonal components demultiplexation by using PBS. Constructed simulation scenarios allows to verify effectiveness of polarization attraction depending on pump input power and SOP as well as length and nonlinearity level of optical fiber. Both co-propagating and counter-propagation configurations have been included in simulation scenarios. As presented, growing pump power and longer fiber enhance effectiveness of polarization attraction, measured by theoretical crosstalk. Co-propagation of pump and probe signal provide several configurations of polarization attractor with crosstalk level lower than $-20 \mathrm{~dB}$, however counter-propagation configurations simulation results present more optimal probe signal DOP characteristics in all range of simulated pump powers. Moreover it is less vulnerable to higher PMDc values. Achieved results are helpful in selecting the best configuration of polarization attractor for optimal polarization control stability, depending on optimization goal. If the goal is to minimize input power level of the pump, there is a possibility of choosing longer fiber, fiber with stronger nonlinearity or different input pump SOP. On the other way, if length of the fiber is to be minimized, one may choose higher pump power and different input SOP. This polarization attractor optimization model may be useful as a step towards construction of polarization demultiplexer for PDM systems. Therefore in the next step of research, achieved simulation results will be verified experimentally. This will be followed by theoretical and experimental verification of effectiveness of the polarization control process for probe signal wavelengths from 1528 to $1560 \mathrm{~nm}$ with the pump at 
$1460 \mathrm{~nm}$. Chosen probe signal wavelength range will allow to verify actual bandwidth of the signal, which polarization could be controlled with a single pump.

Acknowledgements We would like to thank DAWIS IT Sp. z o.o. and European Regional Development Fund for technical support. Grant Number RPMA.01.02.00-14-5632/16.

Open Access This article is distributed under the terms of the Creative Commons Attribution 4.0 International License (http://creativecommons.org/licenses/by/4.0/), which permits unrestricted use, distribution, and reproduction in any medium, provided you give appropriate credit to the original author(s) and the source, provide a link to the Creative Commons license, and indicate if changes were made.

\section{References}

Agrawal, G.: Nonlinear Fiber Optics. Academic Press (2012)

Agrawal, G., Lin, Q.: Vector theory of stimulated Raman scattering and its application to fiber-based Raman amplifiers. J. Opt. Soc. Am. B 20(8), 1616-1631 (2003)

Boyd, R.: Nonlinear Optics. Academic Press, Cambridge (2008)

Chiarello, F., Ursini, L., Palmieri, L., Santagiustina, M.: Polarization attraction in counterpropagating fiber Raman amplifiers. IEEE Photonics Technol. Lett. 23(20), 1457-1459 (2011)

Heebner, J., Bennink, R., Boyd, R., Fisher, R.: Conversion of unpolarized light to polarized light with greater than 50\% efficiency by photorefractive two-beam coupling. Opt. Lett. 25, 257-259 (2000)

Kaminow, I.: Polarization in optical fibers. IEEE J. Quantum Electron. 17(1), 15-22 (1981)

Martinelli, M., Cirigliano, M., Ferrario, M., Marazzi, L., Martelli, P.: Evidence of Raman-induced polarization pulling. Opt. Express 17, 947-955 (2009)

McMaster, W.: Matrix representation of polarization. Rev. Mod. Phys. 33(1), 8-28 (1961)

Morin, P., Pitois, S., Fatome, J.: Simultaneous polarization attraction and Raman amplification of a light beam in optical fibers. J. Opt. Soc. Am. B 29, 2046-2052 (2012)

Muga, N., Ferreira, M., Pinto, A.: Broadband polarization pulling using Raman amplification. Opt. Express 19, 18707-18712 (2011)

Perlicki, K.: Investigation of the state of polarization distribution generated by polarization scramblers on the Poincare sphere. Opt. Commun. 252(1-3), 58-63 (2005)

Perlicki, K.: Impact of an alien wavelength on wavelength division multiplexing transmission quality. Photonics Lett. Pol. 4(3), 118-120 (2012)

Pitois, S., Picozzi, A., Millot, G., Jauslin, H., Haelterman, M.: Polarization and modal attractors in conservative counterpropagating four-wave interaction. Europhys. Lett. 70, 88-94 (2005)

Pitois, S., Fatome, J., Millot, G.: Polarization attraction using counter-propagating waves in optical fiber at telecommunication wavelengths. Opt. Express 16, 6646-6651 (2008)

Rochat, E., Walker, S., Parker, M.: Polarisation and wavelength division multiplexing at $1.55 \mathrm{~mm}$ for bandwidth enhancement of multimode fibre based access networks. Opt. Express 12(10), 2280-2292 (2004)

Thevenaz, L., Zadok, A., Eyal, A., Tur, M.: All-optical polarization control through Brillouin amplification. In: OFC/NFOEC 2008-2008 Conference on Optical Fiber Communication/National Fiber Optic Engineers Conference, pp. 1-3 (2008)

Ursini, L., Santagiustina, M., Palmieri, L.: Raman nonlinear polarization pulling in the pump depleted regime in randomly birefringent fibers. IEEE Photonics Technol. Lett. 23, 254-256 (2011). https://doi. org/10.1109/LPT.2010.2098024 